\title{
INTRASPECIFIC DIVERSITY OF SCORPIONS VENOM AND ITS IMPLICATION IN THE PATHOPHYSIOLOGICAL EFFECTS
}

Thesis: Mohamed A. Abdel-Rahman submitted this thesis for his Doctorate in Zoology (Physiology) at Zoology Department, Faculty of Science, Suez Canal University, Ismailia, Egypt, 2007.

Advisors: Professor Ismail M. Abdel-Nabi, Professor Mohamed Alaa A. Omran, and Professor Alistair McVean

ABSTRACT: The present study was conducted to explore the following hypotheses: (i) do scorpions (the Egyptian scorpion Scorpio maurus palmatus) from different geographical locations exhibit intraspecific diversity in their venom? (ii) if so, is the variation associated with ecological or genetic factors, geographical distance, and/or multiple interrelated factors? and (iii) do the different toxins produce different physiological and toxicological effects to prey? To discover whether there are qualitative differences in the morphological characters and venom composition of $S$. $m$. palmatus, individuals were collected from four geographically isolated localities in Egypt. Three of these locations were from mutually isolated pockets in the arid area of Southern Sinai (Wadi Sahab, El-Agramia and Rahaba plains). The fourth population was sampled from the semi-arid environment of Western Mediterranean Costal Desert (WMCD). The investigation was divided into three main parts. In the first part, we showed that there are morphological differences between geographically separated populations of $S$. m. palmatus and that some aspects of their ecology are distinct. The second part examined intraspecific diversity of scorpion venom (pooled and individual) using SDS-PAGE technique. We also estimated the genetic distance between the four scorpion populations using some molecular techniques (Random Amplified Polymorphic DNA -RAPD, DNA sequence of RAPD markers, and Restriction Fragment Length Polymorphism - RFLP). Finally, we examined the neurotoxic and cytotoxic effects of venom drawn from the four populations on adult cockroach Periplanita americana. Most of the morphometric measurements (total body length, pedipalp length, chela width, number of setae on legs and number of pectinal teeth) revealed highly significant differences among the four populations. Pearson correlation matrix showed an interaction between morphometric measurements and some environmental factors (altitude, soil nature, and climate). This is the first time that a widely distributed scorpion species has been shown to produce a non-uniform cocktail of venom which varies with geographic location. Electrophoresis and a densitometric gel scan showed that pooled venom of WMCD origin contains several protein bands which are absent in Sinai-sourced 
venom. Sinai venom is characterized by the presence of seven bands (150.1$14.8 \mathrm{kDa}$ ) that are not mirrored in WMCD venom. Interestingly, even within each scorpion population there is a clear variation in the venom composition of both males and females. The data of RAPD, RFLP and DNA sequence confirmed the genetic separation among the sampled populations. More than 77 RAPD bands (ranging in size from 100 to 2500bp) were defined from the four scorpion populations using RAPD technique. Of the 77 bands, 57 (76.2\%) were polymorphic and 20 were monomorphic among the populations. The similarity coefficient data of venom and DNA were used to construct separate dendrograms, which revealed that Southern Sinai scorpion populations grouped together and some distanced away from the WMCD population. Our data suggest that RAPD sequences can be used as molecular markers in genetic studies of scorpions. Moreover, our findings revealed that the venom from the different populations exhibited differences in potency. The venom inhibited the activity of acetylcholinesterase followed by spastic paralysis and death in treated insects. We showed that S. m. palmatus venom has the ability to induce oxidative stress to the treated insect groups, manifested by an increase in the level of nitric oxide and protein carbonyl contents, and a decrease in the activities of both $\mathrm{Cu} / \mathrm{Zn}$ superoxide dismutase and catalase.

KEY WORDS: Scorpio maurus palmatus venom, intraspecific diversity, morphology, RAPD, bioinsecticides, Egypt.

\section{CORRESPONDENCE TO:}

MOHAMED A. ABDEL-RAHMAN, Zoology Department, Faculty of Science, Suez Canal University, Ismailia 41522, Egypt. Phone: +20 120862886.

Email: dr moh 71@hotmail.com or mh.abdelrahman@scuegypt.edu.eg. 